\title{
HERMITIAN BILINEAR FORMS WHICH ARE NOT SEMIBOUNDED
}

\author{
BY ALAN MCINTOSH \\ Communicated by Felix Browder, November 5, 1969
}

1. Introduction. Many results from the theory of closed semibounded hermitian bilinear forms in a Hilbert space can be extended so as to apply to forms which are not semibounded and indeed have no restriction placed on their numerical range. Some of these results are summarized in this note, while details can be found in [5]. Our main interest here is in questions which arise naturally when the forms are assumed to be hermitian but not necessarily semibounded. Some such questions are: Is every closed hermitian form $J$ of the type $J_{A}[u, v]=\left(A^{1 / 2} u, A^{1 / 2^{*} v}\right)$ with $A$ selfadjoint? Does every symmetric operator $B$ with equal deficiency indices have a selfadjoint extension $A$ such that the form $J_{A}$ is minimal among those closed hermitian forms whose associated operators extend $B$ ? If such a minimal extension exists, is it necessarily unique?

If our forms and operators are semibounded then each of these questions is known to have a positive answer (see [4, Chapter V]). We will show that in the general case the third question may have a negative answer, and that the second question can be answered in the affirmative when there is a gap in the essential spectrum of $B$. Complete answers to the first two questions are not known to the author.

We remark that one reason for wanting to know whether a closed form is of the type $J_{A}$ is that results from interpolation and spectral theory can then be used to obtain information about the form from our knowledge of the operator $A$.

2. Closed forms. If $X$ and $Y$ are complex linear spaces then a function $J$ from $X \times Y$ to the complex numbers is called a bilinear form (or more properly a sesquilinear form) if it is linear in the first variable and conjugate linear in the second. In the case when $X$ and $Y$ are Hilbert spaces and the bilinear form $J$ is bounded (meaning $|J[u, v]| \leqq \gamma\|u\|_{X}\|v\|_{Y}$ for some positive $\gamma$ and all $u \in X$ and $\left.v \in Y\right)$, there exists a bounded linear operator $C: X \rightarrow Y$ satisfying $J[u, v]$ $=(C u, v)_{Y}$ for $u \in X$ and $v \in Y$. If this operator $C$ is an isomorphism

AMS Subiect Classifications. Primary 4710; Secondary 4615.

Key Words and Phrases. Hilbert space, closed bilinear forms, nonsemibounded hermitian bilinear forms, selfadjoint operators, Friedrichs extensions. 
we say that $J$ is regular.

Throughout this paper $H$ denotes a complex Hilbert space and $J$ a bilinear form with domain $D(J)=X \times Y$ where $X$ and $Y$ are dense linear subspaces of $H$. If $X$ and $Y$ can be given the structure of Hilbert spaces which are continuously embedded in $H$ and if $J$ is regular on $X \times Y$ we say that $J$ is 0 -closed. The bilinear form $J$ is called closed if, for some complex number $\lambda$, the form $J-\lambda$ is 0 closed (where $J-\lambda$ is the form with domain $X \times Y$ defined by $(J-\lambda)[u, v]=J[u, v]-\lambda(u, v))$.

It is shown in [5] that in the case when $X=Y$ and $J$ is semibounded (or regularly accretive) this definition coincides with the usual one (as found for example in [4]). Other results in [5] are:

Proposition 2.1. The operator $A_{J}$ associated with a closed form $J$ has nonempty resolvent set, $\rho\left(A_{J}\right)$. (Here $A_{J}$ is the operator with largest domain satisfying $J[u, v]=\left(A_{J} u, v\right)$.) In fact $\lambda \in \rho\left(A_{J}\right)$ if and only if $J-\lambda$ is 0 -closed, in which case $A_{J-\lambda}=\left(j_{Y}^{*}\right)^{-1} C j_{X}^{-1}$, where $j_{X}: X \rightarrow H$ and $j_{Y}: Y \rightarrow H$ are the continuous embeddings and $C: X \rightarrow Y$ is defined by $(J-\lambda)[u, v]=(C u, v)_{Y}$.

PROPOSITION 2.2. If $A$ is a selfadjoint operator in $H$ with resolution of the identity $\left\{E_{\lambda}\right\}$ then the bilinear form $J_{A}$ with domain $X \times X$ is a closed hermitian form, where $X=\left\{u\left|\int\right| \lambda \mid d\left(E_{\lambda} u, u\right)<\infty\right\}$ and $J_{A}[u, v]$ $=\int \lambda d\left(E_{\lambda} u, v\right)$. Moreover $A=A_{\left(J_{A}\right)}$. We remark that if $A^{1 / 2}$ is a square root of $A$ defined by the operational calculus for selfadjoint operators, then $X=D\left(A^{1 / 2}\right)=D\left(A^{1 / 2^{*}}\right)$ and $J_{A}[u, v]=\left(A^{1 / 2} u, A^{1 / 2^{*} v}\right)$.

By a hermitian form is meant a bilinear form $J$ with domain $X \times X$ such that $J[u, u]$ is real for all $u \in X$. A hermitian form is said to be bounded from below if $J[u, u] \geqq \gamma\|u\|^{2}$ for some real $\gamma$ and all $u \in X$. It is known that every closed bilinear form which is bounded from below is of the type $J_{A}$, but it is not known to the author if this is so for all closed hermitian forms. The following three lemmas are concerned with forms which are of this type. Lemma 2.3 is proved by noting that if $J$ is a closed form with domain $X \times Y$ and associated operator $A_{J}$, then $D\left(A_{J}\right)$ is dense in $X$ [5, Theorem 2.1].

LEMMA 2.3. If $J_{1}$ and $J_{2}$ are two closed bilinear forms with the same domain and the same associated operator, then $J_{1}=J_{2}$. In particular if $J$ is a closed hermitian form with associated operator $A$ and $D(J)$ $=D\left(A^{1 / 2}\right) \times D\left(A^{1 / 2}\right)$, then $J=J_{A}$.

Lemma 2.4. Let $J$ be a closed hermitian bilinear form in $H$, and suppose there is a second inner product $(\cdot, \cdot)^{\prime}$ in $H$ with norm equivalent to the original one. Then $J$ is of the type $J_{A}$ in $H$ if and only if it is of 
the same type with respect to the second inner product.

Proof. On defining $A^{\prime}$ to be the operator associated with $J$ when $H$ has the second inner product, and noting Lemma 2.3, we see that the result will follow once we have shown that $D\left(A^{1 / 2}\right)=D\left(A^{1 / 2}\right)$ (where the square root of $A^{\prime}$ is defined using the second inner product). By interpolation we know that this will be true if $D(A)=D\left(A^{\prime}\right)$ (see [3]). But this follows from the fact that both $D(A)$ and $D\left(A^{\prime}\right)$ are precisely the set $\{u \in X \mid J[u, \cdot]$ is a continuous function on $X$ when taken with the $H$-topology $\}$.

Lemma 2.5. Suppose that $J$ is a closed hermitian bilinear form in $H$ with domain $X \times X$. Then $J$ is of the type $J_{A}$ if and only if there exist closed subspaces $H^{+}$and $H^{-}$of $H$ satisfying:

(i) $H=H^{+} \oplus H^{-}$(where $\oplus$ denotes the algebraic direct sum);

(ii) $X=\left(H^{+} \cap X\right) \oplus\left(H^{-} \cap X\right)$; and

(iii) $J[u, u]>0, J[v, v] \leqq 0$ and $J[u, v]=0$ for all nonzero vectors $u \in H^{+} \cap X$ and $v \in H^{-} \cap X$.

Proof. If $J=J_{A}$ with the selfadjoint operator $A$ having resolution of the identity $\left\{E_{\lambda}\right\}$, then the subspaces $H^{-}=$range $\left(E_{0}\right)$ and $H^{+}=\left(H^{-}\right)^{\perp}$ satisfy the above properties.

Conversely, suppose two subspaces $\mathrm{H}^{+}$and $\mathrm{H}^{-}$exist which satisfy (i), (ii) and (iii). A new inner product with norm equivalent to the original one can be introduced in $H$ by the formula $\left(u_{1}+v_{1}, u_{2}+v_{2}\right)^{\prime}$ $=\left(u_{1}, u_{2}\right)+\left(v_{1}, v_{2}\right)$, where $u_{1}, u_{2} \in H^{+}$and $v_{1}, v_{2} \in H^{-}$. Denote $H$ together with this inner product by $H^{\prime}$ and suppose that the selfadjoint operator $A^{\prime}$ associated with $J$ in $H^{\prime}$ has resolution of the identity $\left\{E_{\lambda}^{\prime}\right\}$. It is not hard to check that $H^{-}=\operatorname{range}\left(E_{0}^{\prime}\right)$. Moreover it can be shown that $J=J_{A^{\prime}}$ (in $H^{\prime}$ ) by noting that the semibounded forms $J^{+}$and $J^{-}$obtained by restricting $J$ to $\mathrm{H}^{+}$and $\mathrm{H}^{-}$respectively are of this type. The result then follows as a consequence of Lemma 2.4 .

3. Existence of minimal closed extensions. A bilinear form $J$ is called (somewhat loosely) an extension of a linear operator $B$ if $A_{J} \supseteq B$. Our interest here is in hermitian extensions of symmetric operators. The following two theorems are direct consequences of results in $\$ 5$ of [5].

THEOREM 3.1. Let $J$ be a closed hermitian extension of a denselydefined operator $B$ (where $D(J)=X \times X$ and $X$ has an appropriate Hilbert space structure). Then $J$ is a minimal closed hermitian extension of $B$ if and only if $D(B)$ is dense in $X$. 
THEOREM 3.2. If $B$ is a symmetric densely-defined operator in $H$ whose closure $\bar{B}$ has finite and equal deficiency indices, then there exists a minimal closed hermitian form $J$ which extends $B$. In fact if $A_{1}$ is any selfadjoint extension of $B$, if the form $J_{A_{1}}$ has domain $X_{1} \times X_{1}$, and if $X$ denotes the closure of $D(B)$ in $X_{1}$, then $J$ can be chosen as the restriction of $J_{A_{1}}$ to the domain $X \times X$.

We now prove that such a form $J$ is necessarily of the type $J_{A}$ under the additional assumption of a "gap" in the essential spectrum of $B$.

To be more precise let $\Delta_{F}(B)$ denote the Fredholm domain of $\bar{B}$ (i.e. the set of complex numbers $\lambda$ for which $\bar{B}-\lambda$ is a Fredholm operator: $\operatorname{cf}[4$, p. 242$]$ ), and let $R$ denote the reals. Then we have:

THEOREM 3.3. If $B$ is a symmetric densely-defined linear operator in $H$ with $\Delta_{F}(B) \cap R \neq \varnothing$, then there exists a selfadjoint operator $A$ for which $J_{A}$ is a minimal closed hermitian extension of $B$.

Proof. Let $A_{1}$ be any selfadjoint extension of $B$, let $J_{1}=J_{A_{1}}$, and let $D(J)=X_{1} \times X_{1}$ (with a suitable Hilbert space structure on $X_{1}$ ). If $X$ is the closure of $D(B)$ in $X_{1}$ then the form $J=\left.J_{1}\right|_{X \times X}$ is a minimal closed hermitian extension of $B$ (as the hypotheses of Theorem 3.2 are satisfied). Let $A=A_{J}$. What must be shown here is that $J$ $=J_{A}$. Now the only spectral points of $A$ in $\Delta_{F}(B)$ are isolated eigenvalues, so $\rho(A) \cap R \neq \varnothing$. It is no restriction to suppose that $0 \in \rho(A)$ (for $B, J_{1}$, etc. can be replaced if necessary by $B-\lambda, J_{1}-\lambda$, etc. where $\lambda \in \rho(A) \cap R)$.

By Lemma 2.5 there exist closed subspaces $H_{1}^{+}$and $H_{1}^{-}$of $H$ which satisfy the properties (i), (ii) and (iii) listed there (with $\mathrm{H}^{+}$and $\mathrm{H}^{-}$ replaced by $H_{1}^{+}$and $H_{1}^{-}$respectively). Now, using the fact that $X$ is a closed subspace with finite codimension in $X_{1}$, it follows that $\left(H_{1}^{-} \cap X\right)$ $\oplus\left(H_{1}^{+} \cap X\right)$ is closed and has finite codimension in $X_{1}$ and hence in $X$ itself. Moreover, there exist closed subspaces $X^{-}$and $X^{+}$of $X$ with $X^{-} \supseteq H_{1}^{-} \cap X, X^{+} \supseteq H_{1}^{+} \cap X$ and $X^{-} \oplus X^{+}=X$, such that if $u$ and $v$ are nonzero elements of $X^{+}$and $X^{-}$respectively we have $J[u, u]>0$, $J[v, v] \leqq 0$ and $J[u, v]=0$. (This can be proved by applying the theory of $J$-spaces to the regular form $J$ in the Hilbert space $X$. See e.g. [2], or [6, Theorem 2.1].)

Now let $\mathrm{H}^{+}$and $\mathrm{H}^{-}$denote the closures in $H$ of $\mathrm{X}^{+}$and $\mathrm{X}^{-}$respectively. These subspaces satisfy properties (i), (ii) and (iii) listed in Lemma 2.5. We conclude that $J=J_{A}$.

4. Uniqueness of minimal closed extensions. It is well known that if a symmetric densely-defined linear operator $B$ is bounded from 
below, then there is a unique minimal closed semibounded bilinear form $J$ which extends $B$. In fact $J$ is the unique minimal closed hermitian form which extends $B$ (for a minimal closed hermitian extension of $B$ is necessarily semibounded). We show by examples that in the case of symmetric operators which are not semibounded, a uniqueness result of this kind sometimes occurs and sometimes does not.

EXAMPLe 4.1. A symmetric operator $B$ with infinitely many minimal closed hermitian extensions $J_{\alpha}$ is obtained in $H=L^{2}(0,1)$ by setting $B u=i(d u / d x)$ with $D(B)=C_{0}^{\infty}(0,1)$. The extensions $A_{\alpha}$ of $B$ defined by $A_{\alpha}=i(d / d x)$ with domains $D\left(A_{\alpha}\right)=\{u \in H \mid u$ is absolutely continuous, $d u / d x \in H$ and $u(1)=\alpha \cdot u(0)\}$ are selfadjoint when $|\alpha|=1$. (For details, see e.g. [1, p. 1305].) For each $\alpha$, let $J_{\alpha}=J_{A \alpha}$ have domain $X_{\alpha} \times X_{\alpha}$ with an appropriate Hilbert space structure on $X_{\alpha}$. By Theorem 3.3, $D(B)$ is dense in at least one of the $X_{\alpha}$. We will show that $D(B)$ is dense in all of the spaces $X_{\alpha}$, and it will follow by Theorem 3.1 that $J_{\alpha}$ is a minimal closed hermitian extension of $B$ for all $\alpha$ (with $|\alpha|=1$ ).

Let $\beta$ be a complex number of absolute value one for which $D(B)$ is dense in $X_{\beta}$, and let $\alpha$ be any other complex number with $|\alpha|=1$. Choose $\gamma$ real so that $\exp (i \gamma)=\alpha \beta^{-1}$, and let $S$ be the unitary operator in $H$ defined by $S u(x)=\exp (i \gamma x) \cdot u(x)$. The operator $S$ is an isomorphism from $D\left(A_{\beta}\right)$ to $D\left(A_{\alpha}\right)$ when $D\left(A_{\beta}\right)$ is normed by $\|u\|_{\beta}^{2}=\|u\|^{2}$ $+\left\|A_{\beta} u\right\|^{2}$ and $D\left(A_{\alpha}\right)$ is normed similarly. (To see this we note that $S$ is a bijection with closed graph, and use the closed graph theorem.) By interpolation (cf. [3]), $S$ is also an isomorphism from $X_{\beta}$ to $X_{\alpha}$. Since $S(D(B))=D(B)$, we deduce that $D(B)$ is dense in $H_{\alpha}$ as was to be shown.

EXAMPLE 4.2. To conclude, we exhibit a closed symmetric operator $B$ which is not semibounded (nor selfadjoint), but which has a unique selfadjoint extension $A$ with the property that $J_{A}$ is a minimal closed hermitian extension of $B$.

Let $A_{1}$ be an unbounded negative selfadjoint operator in a Hilbert space $H_{1}$, and let $B_{2}$ be an unbounded positive closed symmetric densely defined operator in a Hilbert space $H_{2}$ (which is not selfadjoint). Then the linear operator $B=A_{1} \oplus B_{2}$ in the Hilbert space $H=H_{1} \oplus H_{2}$ can be shown to have the required properties. In fact the unique selfadjoint extension $A$ for which $J_{A}$ is minimal is $A$ $=A_{1} \oplus A_{2}$ where $A_{2}$ denotes the Friedrichs extension of $B_{2}$ in $H_{2}$.

REMARK. If a symmetric operator $B$ has a unique selfadjoint extension $A$ for which $J_{A}$ is a minimal closed extension of $B$, it is natural to call $A$ the Friedrichs extension of $B$. We have seen that symmetric 
operators which are not semibounded sometimes have Friedrichs extensions and sometimes do not. It would be of interest to know under what conditions a symmetric operator necessarily has a Friedrichs extension. In particular we could ask: Do elliptic partial differential operators (which are symmetric but not semibounded) necessarily have Friedrichs extensions?

\section{REFERENCES}

1. N. Dunford and J. Schwartz, Linear operators. II: Spectral theory. Selfadjoint operators in Hilbert space, Interscience, New York, 1963. MR 32 \#6181.

2. Ju.P. Ginzburg and I. S. Iohvidov, A study of the geometry of infinite-dimensional spaces with bilinear metric, Uspehi Mat. Nauk 17 (1962), no. 4 (106), 3-56=Russian Math. Surveys 17 (1962), no. 4, 1-51. MR 26 \#2850.

3. T. Kato, A generalization of the Heinz inequality, Proc. Japan Acad. 37 (1961), 305-308. MR 26 \#2876.

4. - Perturbation theory for linear operators, Die Grundlehren der math. Wissenschaften, Band 132, Springer-Verlag, New York, 1966. MR 34 \#3324.

5. A. McIntosh, Bilinear forms in Hilbert space, J. Math. Mech. 19 (1970).

6. R. S. Phillips, On dissipative operators, An AFOSR Scientific Report, Lecture Series in Differential Equations, Session 6, Georgetown University, 1966.

Macquarie University, North Ryde, N.S.W. 2113, Australia 